\title{
Behavior Formula Extraction for Object Trajectory using Curve Fitting Method
}

\author{
Israa Hadi, $\mathrm{PhD}$ \\ Prof., College of Information Technology \\ University of Babylon
}

\author{
Mustafa Sabah \\ Ph.D. Student, \\ AL-Mansour University College
}

\begin{abstract}
One of the main goals of computer vision is to enable computers to replicate the basic functions of human vision such as motion perception and scene understanding. To achieve the goal of intelligent motion perception, much effort has been spent on object tracking, which is one of the most important challenges in computer vision topics.

The formulations of mathematical models of many systems are basic steps in the process of evaluating their behavior; unfortunately, such formulations may become too complex or may not even be possible. Consequently, empirical functional relationships are often developed to describe system behavior using experimental data. Curve fitting, also known as regression analysis, is used to find the "best fit" line or curve for a series of data points. Most of the time, the curve fit will produce an equation that can be used to find points anywhere along the curve.
\end{abstract}

This study proposes new methods to deal with the trajectory by converting the trajectory points into approximation function using curve fitting function to smooth the data; improving the appearance of the trajectory, extracting important features such as slope and intersection point.

\section{General Terms}

Video tracking, numerical analysis, object behavior.

\section{Keywords}

Object tracking, curve fitting, motion trajectory, spatiotemporal.

\section{INTRODUCTION}

Object tracking in video pictures is the essential steps to produce trajectory points of motion object, then presenting the trajectory points in new shapes using curve fitting method in order to obtain trajectory functions, therefore the necessity of discussing in details the curve fitting methods is essential. New studies have been intervened to analyze the object racking in video pictures, especially scholars interested in computer vision, the meaning of object tracking and detection is to create a connection between objects or object parts in consecutive frames and to extract temporal information about objects such as trajectory, posture, speed and direction. However, such process -Tracking detected objects frame by frame in video- creates difficult assignment [11].

In order to understand the proposed method, the study will discuss the curve fitting concept. The purpose of curve fitting is to discuss theoretically the experimental data with a model (function or equation) and to find the parameters linked with this model. Models of primary importance are mechanistic models. Mechanistic models are purposely formulated to supply insight into a chemical, biological, or physical process that is considered to direct the phenomenon under study. Parameters derived from mechanistic models are quantitative estimates of real system properties (rate constants, dissociation constants, catalytic velocities etc.). Therefore the importance to distinguish mechanistic models from empirical models that are mathematical functions formulated to fit a particular curve is essential, but these parameters do not essentially correspond to a biological, chemical or physical property [12].

In fitting data with an approximating function, there are fundamental approaches; the most important involves passing an assumed function (preferably a polynomial) through every data point. However, critics have been expressed towards these approaches represented by that large deviations from a smooth curve (wiggle), an nth-order polynomial for $\mathrm{n}+1$ data points is too complex for large $\mathrm{n}$ values and experimental data are subject to errors and to pass a polynomial through every point is unsuitable. Obviously, these boundaries can be eliminated by using cubic splines [13].

Curve fitting through histories have passed different developments, Sir Francis Galton (1822-1911), a British anthropologist and meteorologist has managed to create regression analysis which is one of curve fitting branches. The term regression was published in Nature in 1885. Galton used the term while talking of his discovery that offspring of seeds "did not tend to resemble their parent seeds in size, but to be always more mediocre [i.e., more average] than they.... The experiments showed further that the mean filial regression towards mediocrity was directly proportional to the parental deviation from it."

Regression Analysis refers to the study of the relationship between a response (dependent) variable, Y, and one or more independent variables, the X's. When this relationship is reasonably approximated by a straight line, it is said to be "linear", and that what is actually nominated as "linear regression". Otherwise, when the relationship follows a curve, it is called as "curvilinear regression". Usually, assuming that the independent variables are measured exactly (without random error), while the dependent variable is measured with random error. Normally, this assumption is not entirely true, but if such assumption is not justified, much more complicated fitting procedure is required. However, if the size of the measurement error in an independent variable is small relative to the range of values of that variable, least squares regression analysis may be used with legitimacy.

In order to relate with a trajectory point as a function, Curve fitting function will be a necessary element of any mathematical interface. The cores advantages of such mathematical interfaces over other methods are that they can be more naturally embody two-dimensional mathematical 
notations. This method of evaluating empirical formulas was developed over a century ago, the procedure involves approximation a function such that the sum of the squares of differences between the approximation function and the actual function [12] [13].

\section{RELATED WORK}

Mathematical model in video Tracking is an important topic in computer vision and it has been studied for several decades. In this section we summarize studies that are related to our proposed tracking work:

Changhyun Choi, Seung-Min Baek and Sukhan Lee [1] have demonstrated that a real-time solution of 3D pose estimation become feasible by combining a fast tracker (such as KLT) with a method of determining the 3D coordinates of tracking points on an object at the time of SIFT based tracking point initiation, assuming that a 3D geometric model with SIFT description of an object is known a-priori. Keeping track of tracking points with KLT, removing the tracking point outliers automatically, and reinitiating the tracking points using SIFT once deteriorated, the 3D pose of an object can be estimated and tracked in real-time. This method can be applied to both mono and stereo camera based 3D pose estimation and tracking. The former guarantees higher frame rates with about $1 \mathrm{~ms}$ of local pose estimation, while the latter assures of more precise pose results but with about $16 \mathrm{~ms}$ of local pose estimation. The experimental investigations have shown the effectiveness of the proposed approach with realtime performance.

Chafik Samir, P.-A.Absil[2] argues In his study expressing the importance to search for an optimizer of the objective function using a steepest-descent method in an adequate set of curves, Chafiq did not try to solve directly the fourth-order differential equation. In the direction, Riemannian manifold M, also addressed the problem of fitting a curve to data points on a closed Riemannian sub manifold of some Euclidean space by means of a Palais-based steepest-descent algorithm applied to the weighted sum of a fitting-related and a regularity-related cost function.

Marcus Baum and Uwe D. Hanebeck[3], expressed clearly about tracking an extended object or a group target, which gives rise to a varying number of measurements from different measurement sources. For this purpose, the shape of the target is tracked in addition to its kinematics. The target extent is modeled with a new approach called Random Hyper surface Model (RHM) that assumes varying measurement sources to lie on scaled versions of the shape boundaries. It is argued that a star-convex RHM is introduced for tracking star convex shape approximations of targets. Bayesian inference for star-convex RHMs is performed by means of a Gaussianassumed state estimator allowing for an efficient recursive closed-form measurement update. Simulations demonstrate the the performance of this approach for typical extended object and group tracking scenarios.

Yang Wang, Simon Lucey[4] extended the constrained local models (CLM) method into the spatio-temporal domain by enforcing the appearance consistency constraint of each local patch between neighboring frames. More importantly, the study shows that the global warp update can be optimized jointly in an efficient manner using convex quadratic fitting so this approach receives improved performance for the task of non-rigid facial motion tracking on the videos of clinical patients.
Roberto Battiti [5] considers the problem of reconstructing curves from sparse and noisy data by using the B-spline curve model. Also consider a least squares formulation of fitting Bsplines, solved by reactive search optimization (RSO) techniques.

Dong Wang, Huchuan $\mathrm{Lu}$ [6] proposed that a generative tracking method based on a novel robust linear regression algorithm. In contrast to existing methods, the proposed Least Soft threshold Squares (LSS) algorithm models the error term with the Gaussian Laplacian distribution, which can be solved efficiently.

\section{LEAST SQUARES METHOD}

Development over the method of evaluating empirical formulas has been occurred over time, such method has been used for many years. In similarly to the method of cubic splines, it attempts to fit a simple function through a set of data points without the wiggle problem associated with highorder polynomials. Unlike the cubic splines technique, it presupposes that the derived functional relationship does not necessarily pass through every data point. The procedure involves approximating a function such that the sum of the squares of the differences between the approximating function and the actual functional values given by the data is a minimum. The basis for the method is represented graphically in figure 1 .

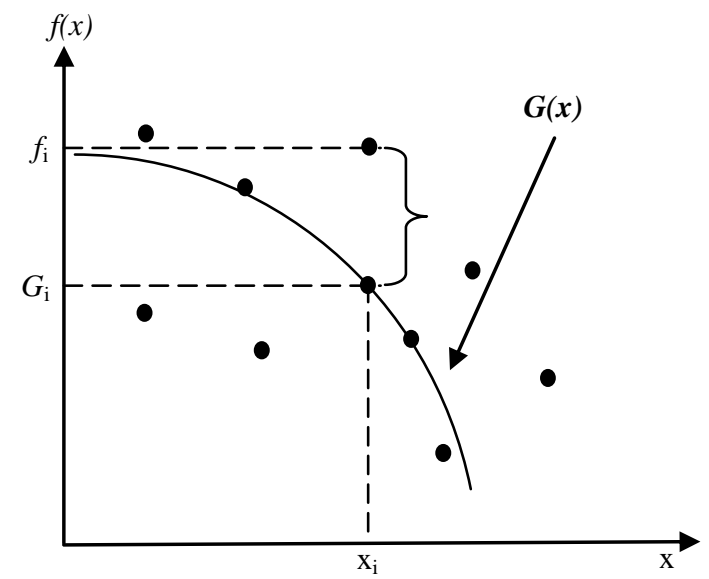

Figure 1: Graphical interpretation of the least-squares method.

Here, given a set of $\mathrm{N}$ data points, to fit an approximating function of the form $\mathrm{G}(\mathrm{x})$ having the following form:

$$
\begin{gathered}
G(x)=a_{1}+g_{1}(x)+a_{2}+g_{2}(x)+\cdots \\
+a_{m} g_{m}(x)
\end{gathered}
$$

Where

$m \leq \operatorname{Nand}_{1}(x), \ldots, g_{m}(x)$ are assumed functions of the independent variable $\mathrm{x}$. The problem is then to evaluate the regression coefficients $a_{1, \ldots, a_{-}}$the method of least squares suggests that these coefficients been easily calculated by minimizing a deviation function $\mathrm{D}$ define as follows:

$D=\sum_{i=1}^{N}\left[f\left(x_{i}\right)-G\left(x_{i}\right)\right]^{2}$ 
Note that since one can fit different $G$ functions through the data set then $a_{1} 1, \ldots, a_{-}$may be looked at as variables. Therefore, using the method of calculus can minimize the function given by equation (2) as shown below:

$$
\begin{aligned}
& \frac{\partial D}{\partial a_{1}}=0 \\
& \frac{\partial D}{\partial a_{2}}=0 \\
& \frac{\partial D}{\partial a_{m}}=0
\end{aligned}
$$

The set of $(\mathrm{m} \mathrm{X} \mathrm{m)} \mathrm{linear} \mathrm{algebraic} \mathrm{equations} \mathrm{given} \mathrm{by}$ equations (3) can therefore be solved for the unknowns $a_{1, \ldots}$ ,$a_{m}$ these coefficients are then substitutes into equation (1) to give the desired approximating function [7].

\section{CURVE FITTING}

In many branches of applied mathematics and engineering sciences experiments and problems come across, which involve two variables. For example, it is known that the speed $v$ of a ship varies with the horsepower $p$ of an engine according to the formula $p=a+b v^{3}$. Here $a, b$ are the constants to be determined. For this purpose several sets of readings of speeds and the corresponding horse powers are taken. The problem is to find the best values for $a, b$ using the observed values of $v$ and $p$.

Thus, the general problem is to find a suitable relation or law that may exist between the variables $x$ and $y$ from a given set of observed values $\left(\mathrm{x}_{\mathrm{i}}, \mathrm{y}_{\mathrm{i}}\right), \mathrm{i}=1,2, \ldots \ldots \ldots . . . n$ Such a relation connecting $x$ and $y$ is known as empirical law, For above example, $x=v$ and $y=p$.

The process of finding the equation of the curve of best fit, which may be most suitable for predicting the unknown values, is known as curve fitting. Therefore, curve fitting means an exact relationship between two variables by algebraic equations. One of the most important aspects of regression (least squares) analysis is related to the question of what functional relationship $\mathrm{G}(\mathrm{x})$ should be assumed. Unfortunately, there are no clear criteria for determining the type of function that can best represent an arbitrary data set. However, the following rules should be observed whenever possible: first plot the data and look for obvious trends such as linear, quadratic, or higher-order behavior. This task can also be accomplished by various steps; the first few differences to see if higher differences tend to zero. If so, then a polynomial approximation may be appropriate. The Second step is to see if the data are symmetrical. Symmetry with respect to $f$ may indicate polynomials of even powers only. Furthermore, symmetry can in some cases be achieved by transforming the data. The Third step considers periodicity. Trigonometric functions may be possible. The Forth steps consider plotting the data on a semi log and/or log-log scale. This may provide information on whether logarithmic or exponential functions can be assumed. Finally, breaking the data set into groups and considering the possibility of assuming different functions for the data subsets.

These rules are helpful in the absence of more suitable criteria, but should be used with discretion. Perhaps the most important rule is that of common sense. That is, if the given data are related to a particular physical phenomenon for which a functional relationship is known, then it makes no sense to assume something different. For example, when examining the relationship between stress and strain for a steel specimen, knowing that the initial portion of this relationship is linear and fully described by Hooke's law. Therefore, if the experimental data indicate nonlinear behavior initially, then the data are in error and a linear function must assume [7] [12] [13].

\section{LINEAR REGRESSION}

Understanding the concept of least squares completely in order to be capable of interpreting the divergence function given by equation (2) it is important to consider figure 2.Given a set of $\mathrm{N}$ data points, a linear function is assumed to exist between the dependent variable $f$ and the independent variable $\mathrm{x}$. That is,

$\mathrm{G}(\mathrm{x})=\mathrm{a}_{1}+\mathrm{a}_{-} \mathrm{x}$

Where:

$$
g_{1}(x)=1 \operatorname{and}_{2}(x)=x .
$$

It is evident that many straight lines can be fitted through any two of the data points. Such an arbitrary procedure will undoubtedly be biased and dependent on individual preference.

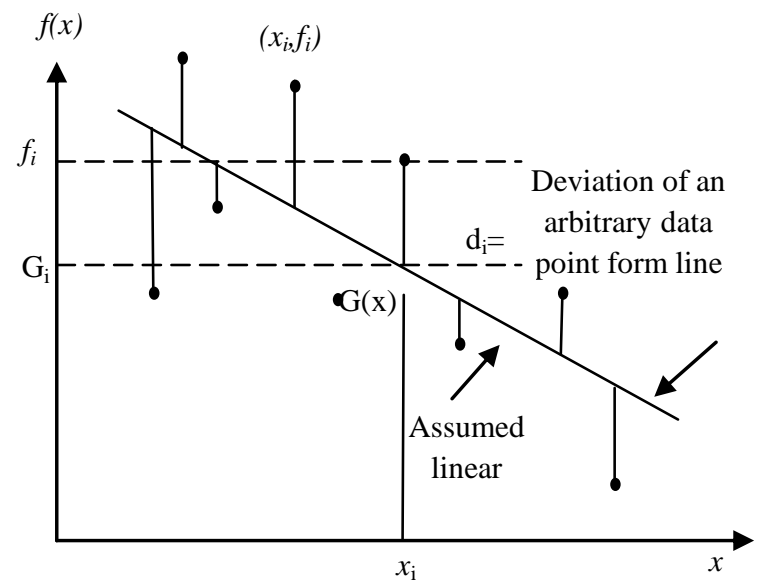

Figure 2: Schematic representation of linear regression.

In fact, there are $\mathrm{S}$ linear equations that can be determined where

$$
S=\frac{N !}{2 !(N-2) !}
$$

Where $\mathrm{N}$ is the number of data points. The implication is that there are $\mathrm{S}$ different sets of values for $\mathrm{a}_{1}$ and $\mathrm{a}_{2}$ that can be evaluated. This implies those $a_{1}$ and $a_{2}$ are indeed variables and must be fixed in such a way that the derived function is the best possible solution to the problem. Such solutions will have the smallest deviations $d_{i}$ from the actual data. The set of deviations is given by:

$$
\begin{aligned}
& d_{1}=f_{1}-G\left(x_{1}\right)=f_{1}-a_{1}-a_{2} x_{1} \\
& d_{2}=f_{2}-G\left(x_{2}\right)=f_{2}-a_{1}-a_{2} x_{2} \\
& \vdots \\
& d_{i}=f_{i}-G\left(x_{i}\right)=f_{i}-a_{1}-a_{2} x_{i} \\
& \vdots \\
& d_{N}=f_{N}-G\left(x_{N}\right)=f_{N}-a_{1}-a_{2} x_{N}
\end{aligned}
$$


The object is to minimize the sum of all of the deviations. Unfortunately, this is not possible because the negative deviations will reduce the positive ones. However, if the sum of the squares of the deviations is determined then a true estimate of the total deviation is possible. This is precisely what equation (2) states. Consequently for an assumed linear approximation we have:

$d^{2}=D=\sum_{i=1}^{N}\left[f\left(x_{i}\right)-G\left(x_{i}\right)\right]^{2}=\sum_{i=1}^{N}\left(f_{i}-a_{1}-a_{2} x_{i}\right)^{2}$

Note that with respect to $a_{1}$ and $a_{2}$ then setting the resulting expressions to zero gives:

$$
\begin{gathered}
\frac{\partial D}{\partial a_{2}}=0=2 \sum_{i=1}^{N}\left(f_{i}-a_{1}-a_{2} x_{i}\right)\left(-x_{i}\right) \\
\frac{\partial D}{\partial a_{1}}=0=2 \sum_{i=1}^{N}\left(f_{i}-a_{1}-a_{2} x_{i}\right)(-1)
\end{gathered}
$$

Simplifying and rearranging yields the following set of $2 \times 2$ linear algebraic equations in the unknown's $\mathrm{a}_{1}$ and $\mathrm{a}_{2}$ :

$$
\begin{aligned}
& \sum_{i=1}^{N} f_{i}=a_{1} N+a_{2} \sum_{i=1}^{N} x_{i} \\
& \sum_{i=1}^{N} f_{i} x_{i}=a_{1} \sum_{i=1}^{N} x_{i}+a_{2} \sum_{i=1}^{N} x_{i}{ }^{2}
\end{aligned}
$$

Equation (5) can therefore be solved to give

$$
\begin{aligned}
& a_{1}=\frac{\sum f_{i} \sum x_{i}^{2}-\sum x_{i} \sum f_{i} x_{i}}{N \sum x_{i}{ }^{2}-\left(\sum x_{i}\right)^{2}} \\
& a_{2}=\frac{N \sum f_{i} x_{i}-\sum x_{i} \sum f_{i}}{N \sum x_{i}{ }^{2}-\left(\sum x_{i}\right)^{2}}
\end{aligned}
$$

Note that for simplicity the limits have been omitted from the sums in equation (6). It is evident that the assumed linear functional relationship is now completely defined in terms of the given data set [2] [7].

\section{LINEARIZATION}

Regularly, the performance exhibited by a certain data set may appear to be nonlinear. Nevertheless, in certain cases the assumed nonlinear approximating function can be changed into a linear directly operating on the data, emerging advantageous as it allows using equations (6) to achieve a solution. For illustration purposes, suppose that given data $\left(x_{i}, f_{i}\right)$ exhibit behavior that can be best described by following function:

$$
G(x)=a_{1} x^{a 2}
$$

Equation (7) can easily be transformed to an equivalent linear form by simply taking the natural logarithm of both sides (obviously, taking $\log 10$ will work just as well). Thus:

$\ln G=\ln a_{1}+a_{2} \ln x$

Now making the following substitutions into equation (7),

$$
\begin{aligned}
& \bar{G}=\ln G \\
& \bar{a}_{1}=\ln a_{1} \\
& \bar{x}=\ln x
\end{aligned}
$$

$\bar{f}=\ln f$

The equivalent linear functional relationship is to that, given by equation (7). That is,

$\bar{G}=\bar{a} 1+a_{2} \bar{x}$

Comparison of equation (8e) to equation (4) clearly shows that they are equivalent. Consequently, their solution must be equivalent as well. Hence, using equation (6), we have

$\bar{a}_{1}$

$=\frac{\sum \overline{f_{i}} \sum \overline{x^{2}}{ }_{i}-\sum \bar{x}_{i} \sum \overline{f_{i}} \overline{x_{i}}}{N \sum \overline{x^{2}}{ }_{i}-\left(\sum \overline{x_{i}}\right)^{2}}$

$a_{2}$

$$
=\frac{N \sum \bar{f}_{i} \bar{x}_{i}-\sum \bar{x}_{i} \sum \bar{f}_{i}}{N \sum \bar{x}_{i}^{2}-\left(\sum \bar{x}_{i}\right)^{2}}
$$

Then, once $\bar{a}_{1}$ and $\bar{a}_{2}$ are determined, equation (8b) is solved for $a_{1}$ and then substituted into equation (7). It is evident that similar transformations are possible for functions of the following form:

$G(x)=a_{1} e^{a_{2} x}$

For cases in which the given data involve zeros or negative numbers, one must transform the data to an equivalent set in which all values are positive. This may be accomplished by adding an arbitrary scalar and introducing a set of new variables, in some applications the assumed functional relationship may be given in the form

$G(x)=a_{1} x^{a_{2}}+c$

Where, $\mathrm{c}$ is a known constant. Such problems can easily be handled by subtracting the constant $\mathrm{c}$ from both sides to give

$G(x)-c=a_{1} x^{a_{2}}$ Then introducing the transformation $T(x)=G(x)-c$

Yields the modified form $T(x)=a_{1} x^{a_{2}}$

Obviously, this equation is of the same form as equation (7) and can be transformed in a similar manner [5] [13].

\section{NON LINEAR REGRESSION}

Assuming that functional approximation is not considered as nonlinear, and linearization in not possible, the regression coefficients must be established by utilizing the deviation function. For example, suppose that the data $\left(x_{i}, f_{i}\right)$ suggest a second-order polynomial of the form

$G(x)=a_{1}+a_{2} x+a_{3} x^{2}$

Where $g_{1}(x)=1, g_{2}(x)=x, g_{3}(x)=x^{2}$.

The corresponding deviation function that must be minimized with respect to the unknown regression coefficients is given by equation (2) as following:

$$
D=\sum_{i=1}^{N}\left(f_{i}-a_{1}-a_{2} x_{i}-a_{3} x^{2}{ }_{i}\right)^{2}
$$

Consequently, differentiating with respect to $a_{1}, a_{2}$ and $a_{3}$ yields the following three linear algebraic equations: 


$$
\begin{gathered}
\frac{\partial D}{\partial a_{1}}=0=2 \sum_{i=1}^{N}\left(f_{1}-a_{1}-a_{2} x_{i}-a_{3} x_{i}^{2}\right)(-1) \\
\frac{\partial D}{\partial a_{2}}=0=2 \sum_{i=1}^{N}\left(f_{1}-a_{1}-a_{2} x_{i}-a_{3} x^{2}{ }_{i}\right)\left(-x_{i}\right) \\
\frac{\partial D}{\partial a_{3}}=0=2 \sum_{i=1}^{N}\left(f_{1}-a_{1}-a_{2} x_{i}-a_{3} x_{i}^{2}\right)\left(-x^{2}{ }_{i}\right)
\end{gathered}
$$

Simplifying and rearranging yields the following more convenient form:

$$
\begin{aligned}
& \sum_{i=1}^{N} f_{i}=a_{1} N+a_{2} \sum_{i=1}^{N} x_{i}+a_{3} \sum_{i=1}^{N} x^{2}{ }_{i} \\
& \sum_{i=1}^{N} f_{i} x_{i}=a_{1} \sum_{i=1}^{N} x_{i}+a_{2} \sum_{i=1}^{N} x^{2}{ }_{i}+a_{3} \sum_{i=1}^{N} x^{3}{ }_{i}
\end{aligned}
$$

$\sum_{i=1}^{N} f_{i} x^{2}{ }_{i}=a_{1} \sum_{i=1}^{N} x^{2}{ }_{i}+a_{2} \sum_{i=1}^{N} x^{3}{ }_{i}+a_{3} \sum_{i=1}^{N} x^{4}{ }_{i}$

Consequently, solving equation (11) for the unknowns $a_{1}, a_{2}$ and $a_{3}$, then substituting into equation (10) yields the desires approximating regression polynomial. It is evident that the procedure just outlined can be easily extended to highorder polynomials or even transcendental functions. Unfortunately, the resulting set of linear algebraic equations in the unknown regression coefficients tends to become unstable as the order of the assumed polynomial increases [7] [12].

\section{TRAJECTORY}

Generally, spatio-temporal trajectories can be classified into two major categories, according to the nature of the underlying spatial object: (i) objects without area represented as moving points, and (ii) objects with area, represented as moving regions; in this case the region extent may also change with time. Among the above two categories, the former has attracted the main part of the research interest, since the majority of the real-world applications involving spatio-temporal trajectories consider objects represented as points, e.g., fleet management systems monitoring cars in road networks [8]. Figure (3) hereinafter, explains the spatiotemporal trajectory of a moving point, where dots represent sampled positions and lines in between represent alternative interpolation techniques (linear vs. arc interpolation). Unknown type of motion can be also found in a trajectory (see $[\mathrm{t} 3, \mathrm{t} 4])$ time interval).

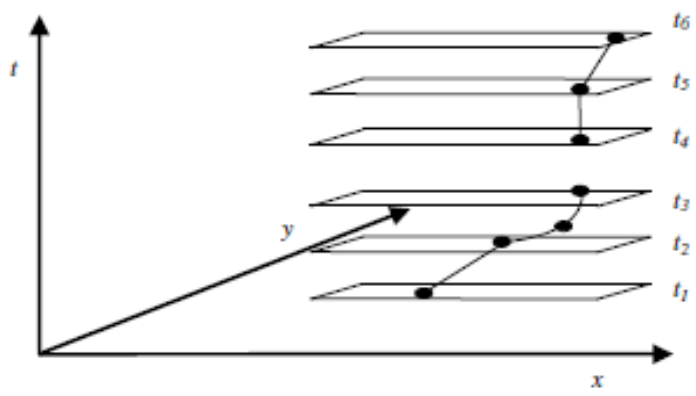

Figure 3: Spatio-temporal Trajectory
The first and foremost restriction posed by such spatiotemporal interpolation methods, is that a trajectory connected to a data sample should contain the sample points. i.e., for all points $\left.x_{i}, y_{i}, t_{i}\right)$ in the sample it holds that :

$\left(\mathrm{x}_{\mathrm{i}}, \mathrm{y}_{\mathrm{i}}, \mathrm{t}_{\mathrm{i}}\right)=\left(\mathrm{a}_{\mathrm{x}}\left(\mathrm{t}_{\mathrm{i}}\right), \mathrm{a}_{\mathrm{y}}\left(\mathrm{t}_{\mathrm{i}}\right), \mathrm{t}_{\mathrm{i}}\right)$. Secondly, given a data sample, there are an infinite number of trajectories connected to that data sample, which implies that the trajectory is by no means unique. Finding a suitable curve connecting the sample points is called interpolation.

Interpolation brings along its own problems; it is required to be fast, easily manageable, flexible and accurate. Unfortunately improving one property doesn't necessarily improve another. Linear interpolation is the fastest and easiest of them all as shown in figure (4). The idea is to connect the sample points with straight lines; the linearity is expressed in the fact that equal jumps in time (between the same sample points) lead to equal jumps in space [9].

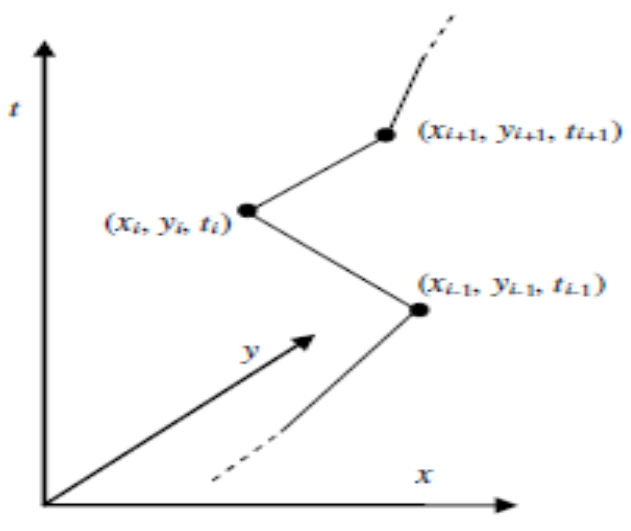

Figure 4: Linear interpolation

Linear interpolation in this manner is not so innocent; along the way some assumptions have been made. The first one is that the moving object keeps constant speed and direction between the sample points. Moreover, this speed is the average speed needed to cover the distance between $\left(\mathrm{x}_{\mathrm{i}}, \mathrm{y}_{\mathrm{i}}\right)$ and $\left(\mathrm{x}_{\mathrm{i}+1}, \mathrm{y}_{\mathrm{i}+1}\right)$ in time $t_{i+1}-t_{i}$. Secondly, changes in speed and direction at sample points are often abrupt and discontinuous, due to the sharp corners of the trajectory at the sample points. On the other hand, linear interpolation is fast to construct and to handle, and this is the main reason why it has been widely adopted in the trajectory database literature.

Among the variety of technologies involved in the development of Moving Object Databases (MODs) for supporting historical trajectories of moving points[14].

\section{PROPOSED APPROACH}

In order to overcome the disadvantages of the related works discussed above, proposing a new presentation method that combines trajectory with curve fitting approach in order to represent all trajectories' points with suitable function.

This proposed approach has many advantages such as storage file reduction, complexity reduction, since this research focuses to deal with trajectories as one parameter, aiming to facilitate video analysis for tracking purposes and to understand the object behavior.

Mathematical equations contain information in densely packed form. That is the single most important reason why data is often subjected to the process of curve fitting. This new combination method leads to Storage efficiency 
throughout storing and managing data that consumes the least amount of space, with little rather than no impact on performance; resulting in a lower total operational cost therefore capacity optimization takes place.

The combination method Often better for extrapolation and differentiation, Parameter values are obtained after the process of deciding on a curve form and linearization, and Parameter values which give the minimum sums of squares for the linear equation do not give minimum sums of squares for the un linear, or original form of the equation. The objective of this papers is to construct a novel framework and understanding for video analysis, this method extract the behavior from trajectory function .after Build a formulation of mathematical models for several points (each set correspond to object motion) in order to evaluate their behavior by fitting data with an approximation function.

This study concentrates to develop new idea based on mixed of more than one methods of numerical analysis, in order to build a map of common paths, each object is recorded with list of data for each frame but in this paper each object trajectory represent by one equation. Figure (5) below explains different available data set (trajectory), such figure have been extracted from various papers and applications to explain how to build "trajectory function".

There are some consideration in choosing a suitable function, Exponential function cannot pass through the origin and can fit only data with all positive y's or all negative y's. Logarithmic function cannot model $\mathrm{x}=0$ or negative value of $\mathrm{x}$ while for power function, $\mathrm{y}=0$ when $\mathrm{x}=0$ finally the reciprocal equation cannot model $\mathrm{y}=0$.

Finally, the proposed method can be considered as a new presentation method that could converts the shape of a trajectory from a list of huge points into one equation, representing approximately all points.

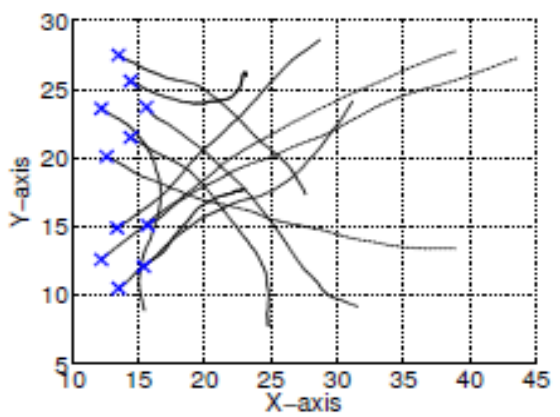

(a)

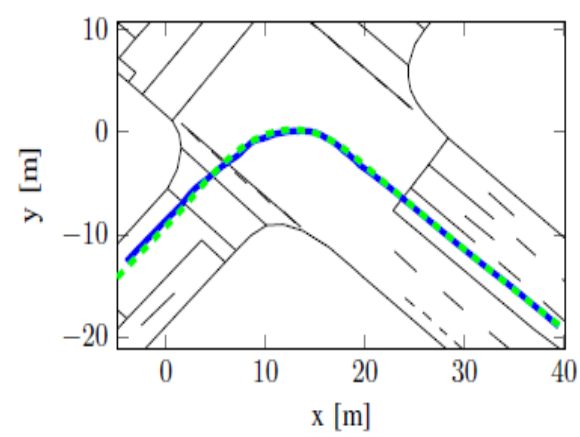

(b)

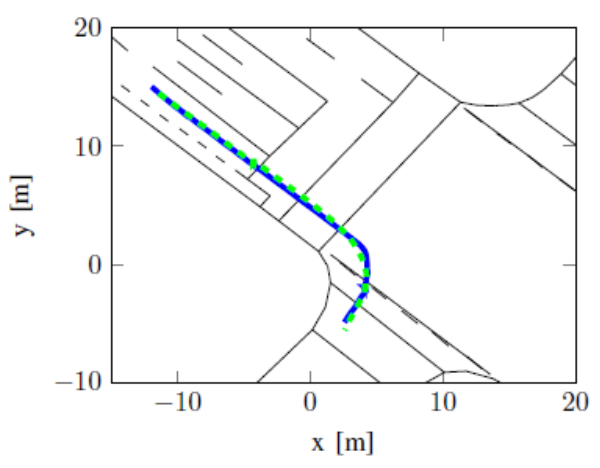

(c)

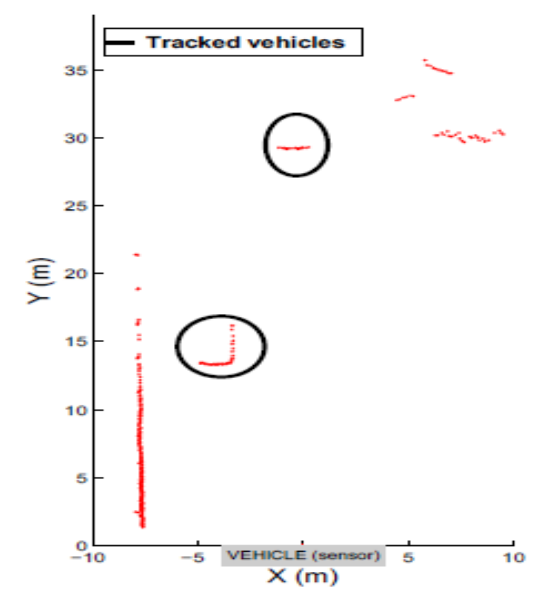

(d)

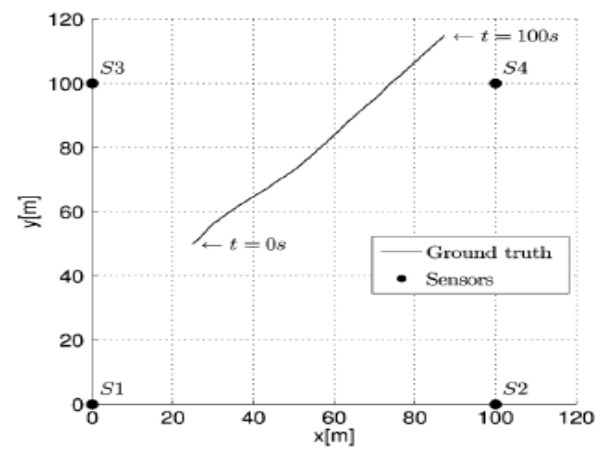

(e)

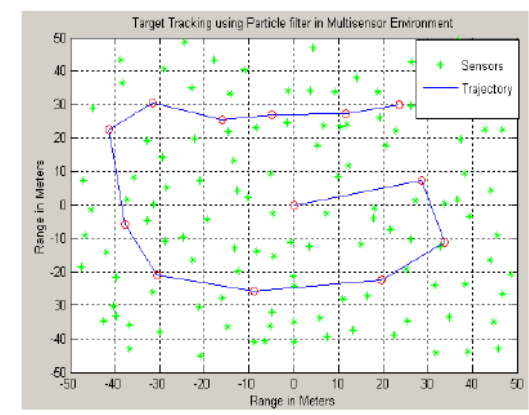

(f)

Figure 5: Different data sets (trajectories) 


\section{EXPERIMENTAL RESULTS}

Experiments were performed over the trajectory dataset to convert the shape of trajectory points into trajectory function; such method can be considered a new nomination in computer vision field.

It is important to reveal that such data was collected from previous resources; the experiments contained 6 data sets.

Regarding the first experiment, the result that contains more trajectories points and cloud view were deployed; such method is to justify the evaluation and behavior of all trajectories.

After applying the curve fitting method to figure 5(a), and transferring all points into trajectories function as shown in approximation equations $12,13,14,15,16,17$ below:

$$
\begin{aligned}
& G(x)=37.6-0.7 x \\
& G(x)=37.2-1.3 x+0.03 x^{2} \\
& G(x)=38.9-0.9 x \\
& G(x)=199.8-24.2 x+0.8 x^{2} \\
& G(x)=-0.02 \ln (x)+24.8 \\
& G(x)=0.05 \ln (x)+8.2
\end{aligned}
$$

Figure 6 (a), (b), (c), (d), (e), (f) shows the cumulated trajectories function, noting that all results have been implemented in Matlab.

The remain experiments were performed over 5 trajectories, to evaluate the behavior for multiple objects tracking, applying different shapes of function and to form different analysis of events, taking the data sets of figure $5(\mathrm{~b})(\mathrm{c})(\mathrm{d})(\mathrm{e})(\mathrm{f}), \quad$ transferring all points into trajectories function as shown in equations 18, 19, 20,21,22,23 below:

$G(x)=-8.2+1.2 x-0.05 x^{2}+0.0003 x^{3}$

$G(x)=2.06-2.07 x+0.08 x^{2}+0.02 x^{3}$

$G(x)=18.9 e^{0.08 x}$

$\left.G(x)=18.9 \mathrm{e}_{(-5.4 *(10)}{ }^{-16}\right)$

$G(x)=1 /-1.7 * 10^{-4} x+0.02$

$G(x)=5.7-0.05 x-0.0002 x^{2}$

Figure $6(\mathrm{~g}),(\mathrm{h}),(\mathrm{i}),(\mathrm{j}),(\mathrm{k}),(\mathrm{l})$ shows the cumulated trajectories function. From the results, different trajectories function can be built, this method could lead to data reduction, complexity reduction, discovering the missing data, and simplifying video analysis.

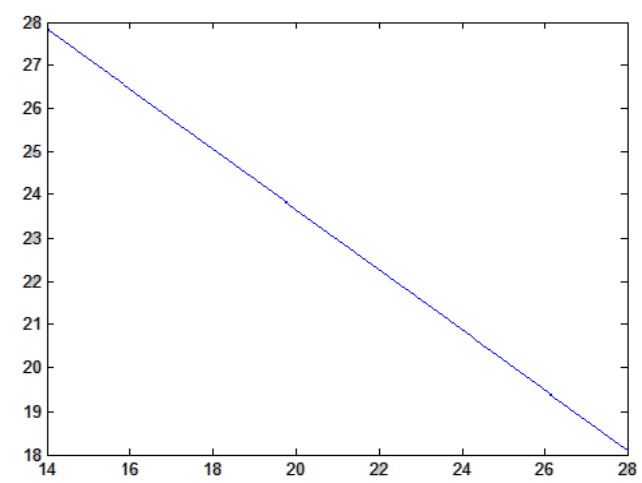

(a)

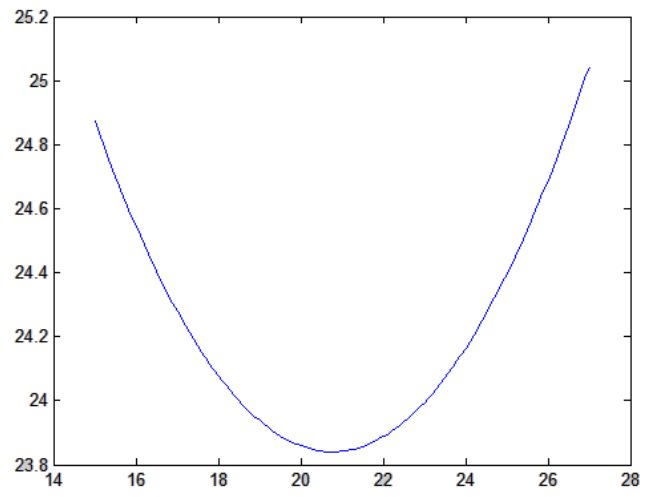

(b)

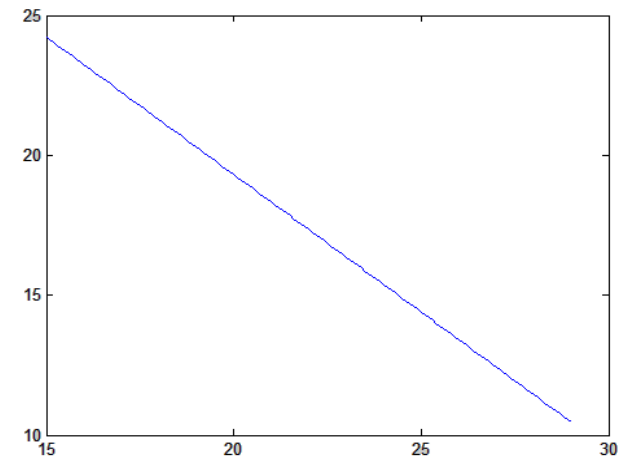

(c)

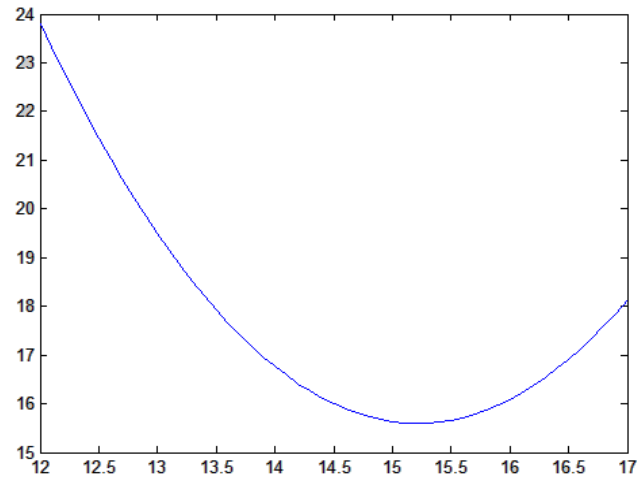

(d)

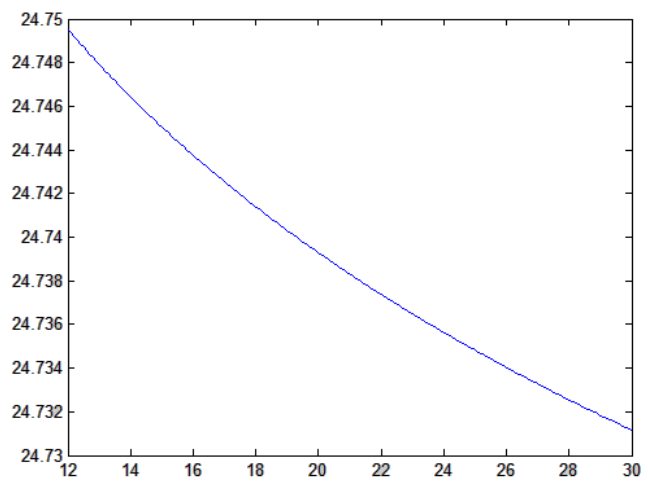

(e) 


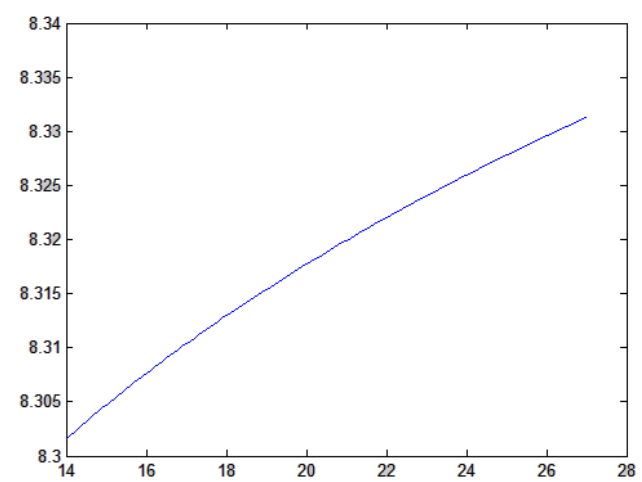

(f)

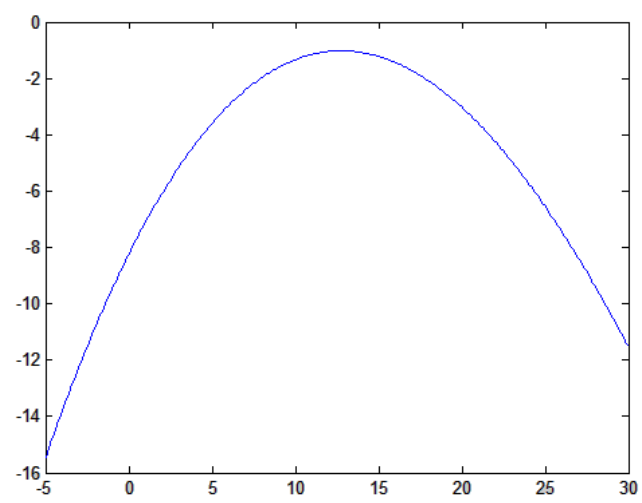

(g)

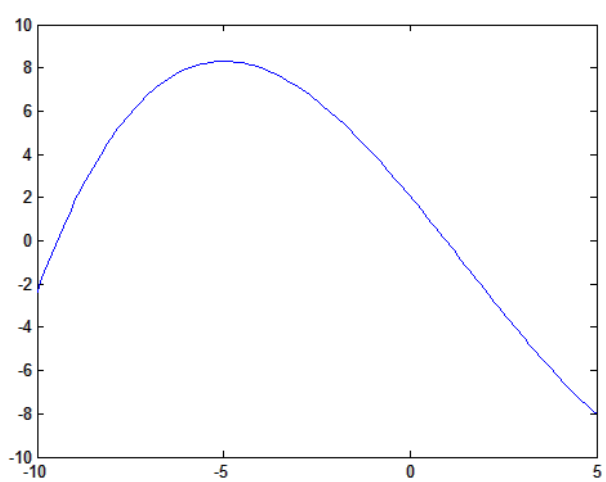

(h)

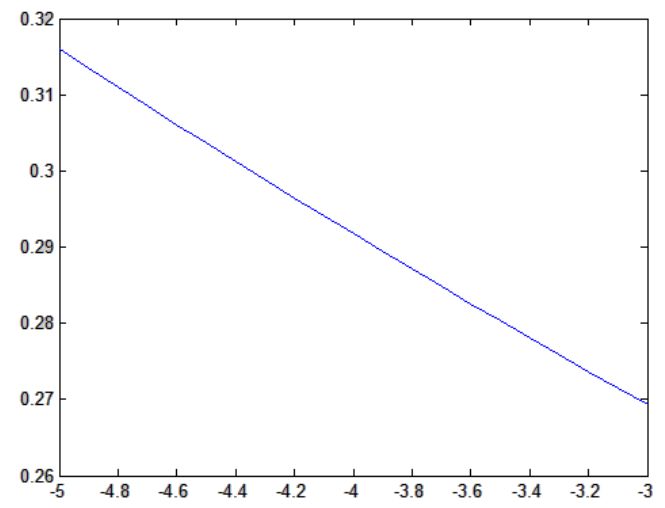

(i)

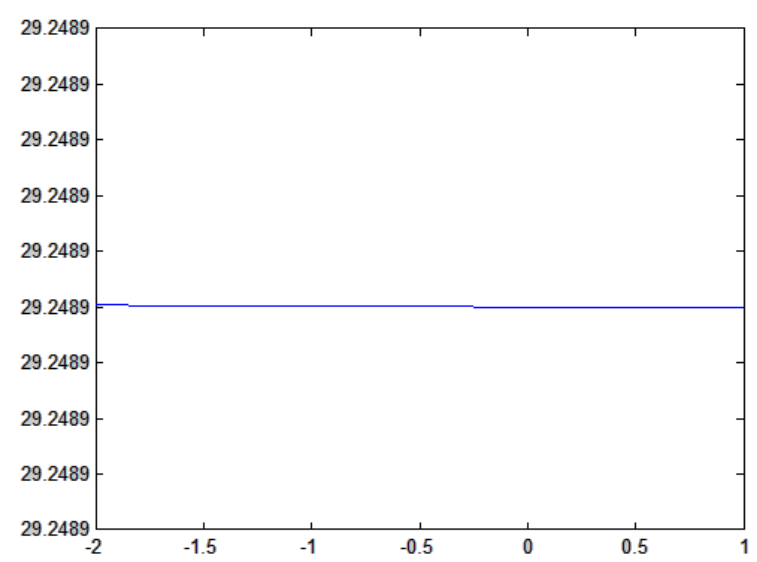

(j)

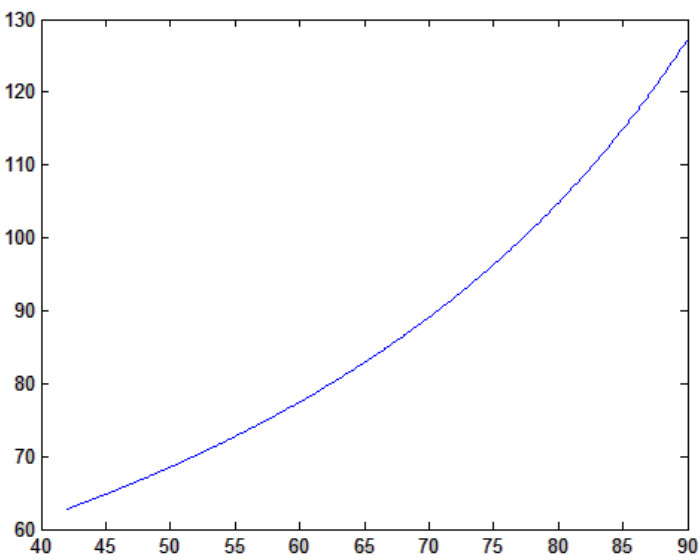

(k)

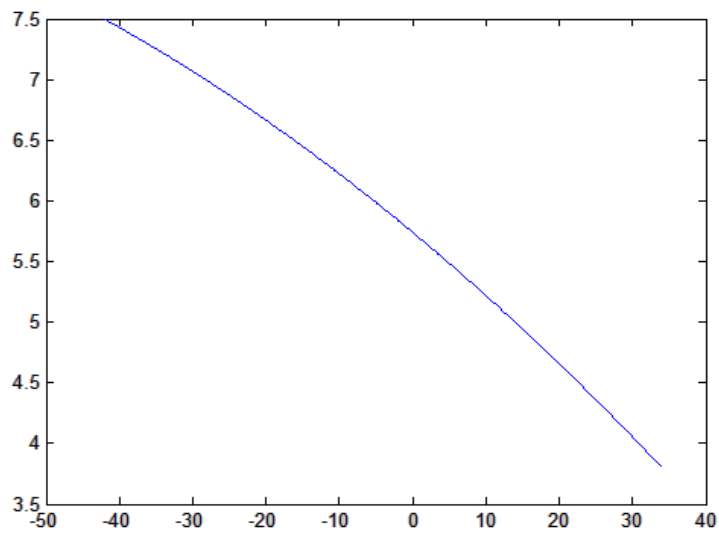

(1)

Figure 6: Trajectory functions corresponding to trajectory points

\section{COMPARISON AND EVALUATION}

This study has introduced a computationally efficient method for trajectory representation by addressing various main challenges, such as: storage file size, missing data due to huge points, and complexity. The proposed method introduced represents a method where the history of all data sets is maintained; in addition, such method will enable the future estimation of new points of trajectory motion object. 
Data on documents (PND) is the first method of performance criteria that could be used for complexity estimation Summation of different data types gives a number which represents a system complexity. This method statistically correlates with referent measuring method FP. PND could very fast accomplish system complexity estimation, and foresee measuring results by using FP method.

The second performance criteria could be represented by Trajectory information, the later refers to the Time Space Position Information (TSPI). It gives positional information, velocity and acceleration of the target at a particular time in space. Behavioral information includes the pitch, yaw and roll rate. Another significant parameter is the maximum range coverage and agreement of the trajectory with the nominal. Measurement of miss-distance between two targets is also very important parameter for the evaluation.

There are differences in trajectory behavior can be quantified in a number of ways. Measuring the trajectory complexity is trajectory arc length (or path length) the length of a path of a over some time interval $T$.

The table (1) clarifies the comparison between Trajectory Points and the proposed method the core of this study (Trajectory Function)

\begin{tabular}{|l|l|l|}
\hline Performance Criteria & $\begin{array}{l}\text { Trajectory } \\
\text { points }\end{array}$ & $\begin{array}{l}\text { Trajectory } \\
\text { function }\end{array}$ \\
\hline Accuracy & Real points & $\begin{array}{l}\text { Approximate } \\
\text { points }\end{array}$ \\
\hline Availability & $\begin{array}{l}\text { Missing } \\
\text { some points } \\
\text { (according } \\
\text { to obstacles) }\end{array}$ & $\begin{array}{l}\text { All points are } \\
\text { available }\end{array}$ \\
\hline PND & Huge & Normal \\
\hline Trajectory complexity & Large & Simple \\
\hline TP & 116 & 100 \\
\hline
\end{tabular}

Table 1: performance evaluation

The points represented in the figure (7) below refer to the normal trajectory points of multiple motion objects, while the continuous line represents the proposed Trajectory Function. It is important to note the following:

1- There are missing data in trajectory points, while such data exists completely in Trajectory Function.

2- In Trajectory Function, estimating new point of motion object can be achieved, while such estimation is unreachable in Trajectory points.

3- A large file is required to save the numerous point in Trajectory points, while such size of file is not required Trajectory Function.

4- One equation can be enough to represent the massive data in trajectory function, while it is impossible to utilize one equation to represent data in Trajectory Point

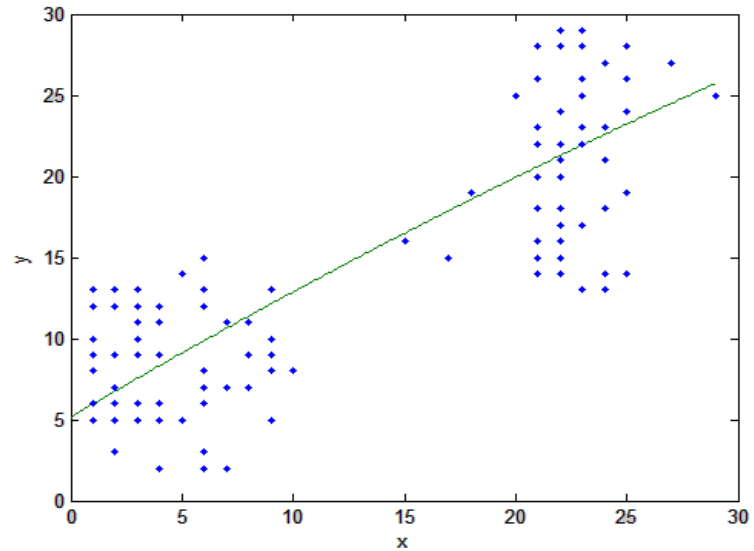

Figure 7: Trajectory functions and trajectory points corresponding to multiple motion objects

\section{CONCLUSION}

Several studies have focused on the semantic properties of trajectories. All of them deal with trajectory as a set of points this assumption leads to huge data and increase the storage file and increase overall overhead and complexity. The novelty of this paper is the creation "trajectory function" for each object in sequence of frames that make able to build knowledge base to infer new and more knowledge about important places of trajectories.

In this paper fit data was used for the purpose of built-in and defining: fitting functions, linear, polynomial and non-linear regression, virtually unlimited number of fit coefficients in user-defined fitting functions have been deployed, automatic calculation of the model curve, curve fit residuals, and confidence and prediction bands have been explained. These curves can be automatically added to a graph of available data. The study infers to the behavior of moving object and understands the goals of trajectory.

The experimental results validate solutions by showing the usefulness of using numerical analysis with video tracking and improvement in performance compared to trajectory points approach. The results demonstrated that the proposed method is more robust to noise and to missing object observations. Future work is focused on event modeling using trajectory mining depend on some important features that can be extracted from trajectory function and represented in $3 \mathrm{D}$ shapes.

\section{REFERENCES}

[1] Changhyun Choi, Seung-Min Baek and Sukhan Lee, "Real-time 3D Object Pose Estimation and Tracking for Natural Landmark Based Visual Servo", IEEE/RSJ international conference, Pages 3983-3989, 2008.

[2] Chafik Samir, P.A. Absil, AnujSrivastava, Eric Klassen, "A Gradient-Descent Method for Curve Fitting on Riemannian Manifolds",paper presents research results of the Belgian Network DYSCO (Dynamical Systems, Control, and Optimization), funded by the Interuniversity Attraction Poles Programmer, initiated by the Belgian State, Science Policy Office. The scientific responsibility rests with its authors. This research was supported in part by AFOSR FA9550-06-1-0324 and ONR N00014-0910664.March 9, 2011 
[3] Marcus Baum, Uwe D. Hanebeck," Shape Tracking of Extended Objects and Group Targets with Star-Convex RHMs", 14th IEEE international conference, Pages 1-8, 2011.

[4] Yang Wang, Simon Lucey, Jeffrey F. Cohn, Jason Saragih," Non-rigid Face Tracking with Local Appearance Consistency Constraint", 18th IEEE International conference, Pages 3045-3048, 2008.

[5] Roberto Battiti," Reconstructing Curve from Sparse and noisy data through reactive search optimization techniques", master thesis, 2009.

[6] Dong Wang, Huchuan Lu, Ming-Hsuan Yang," Least Soft threshold Squares Tracking ",IEEE international conference, Pages 2371-2378, 2013.

[7] Steven "numerical method for engineering", Wiley, 2006

[8] S. J. Rajput, S.D.Oza, "A New Algorithm for Tracking of Multiple Moving Objects" international Journal of Scientific Engineering and Technology, Volume No.2, Issue No.7, pp : 691-693,2013.

[9] Xi Li, Zhang, shen "surveys of appearance models in visual object tracking" ACM transactions on technology, 2013.
[10] Elias K.," Trajectory Data Management in Moving Object Databases", PhD Thesis, Department of Informatics, University of Piraeus, 2008.

[11] Wei Qu, Faisal I. Bashir, Dan Graupe, AshfaqKhokhar, and Dan Schonfeld,"A Motion Trajectory Based Video Retrieval System Using Parallel Adaptive Self Organizing Maps", IEEE international conference, Pages 1800-1805, 2005.

[12] Press, W. H., Flannery, B. P., Teukolsky, S. A., and Vetterling, W. T. Numerical Recipes in C. Cambridge University Press, New York (1988).

[13] Chambers, J. M., Cleveland, W. S., Kleiner, B., and Tukey, P. A. Graphical Methods for Data Analysis. Duxbury Press, Boston (1983).

[14] Pedro Carvalho,Telmo Oliveira ,Lucian Ciobanu," Analysis of object description methods in a video object tracking environment", Springer-Verlag Berlin Heidelberg, Volume 24, Issue 6, pp 1149-1165, 2013.

[15] Uwe Jaenen, Udo Feuerhake, Tobias Klinger," Improving the Quality of object tracking using self organization camera networks", XXII ISPRS Congress, Melbourne, Australia, Volume I-4, 2012.

[16] Bastian Leibe, Konrad Schindler," Coupled Detection and Trajectory Estimation for Multi-Object Tracking", 11th IEEE international conference, Pages 1-8, 2007. 